\title{
Analysis of fractional multi-dimensional Navier-Stokes equation
}

\author{
Yu-Ming Chu ${ }^{1,2}$, Nehad Ali Shah ${ }^{3,4^{*}}$, Praveen Agarwal ${ }^{5}$ and Jae Dong Chung ${ }^{6}$
}

\section{"Correspondence:}

nehad.ali.shah@tdtu.edu.vn

${ }^{3}$ Informetrics Research Group, Ton

Duc Thang University, Ho Chi Minh

City, Vietnam

${ }^{4}$ Faculty of Mathematics \& Statistics,

Ton Duc Thang University, Ho Chi

Minh City, Vietnam

Full list of author information is

available at the end of the article

\section{空 Springer}

\begin{abstract}
In this paper, a hybrid method called variational iteration transform method has been implemented to solve fractional-order Navier-Stokes equation. Caputo operator describes fractional-order derivatives. The solutions of three examples are presented to show the validity of the current method without using Adomian and He's polynomials. The results of the proposed method are shown and analyzed with the help of figures. It is shown that the proposed method is found to be efficient, reliable, and easy to implement for various related problems of science and engineering.
\end{abstract}

Keywords: Variational iteration method; Laplace transform; Caputo derivatives; Navier-Stokes equations

\section{Introduction}

Navier-Stokes equations (NSEs) depicting the physical interests of engineering and scientific research are viewed as beneficial. These equations are predominantly used to manage climate estimating, sea flows, water stream in a line, and wind current around a wing. Also, the basic plan of airplanes and vehicles, the investigation of the bloodstream, the goal of intensity stations, and the examination of contamination are firmly identified with NSEs. Moreover, the study of magnetohydrodynamics depends on the coupling of Maxwell's and NSEs. Since their presentation, distinctive physical models have been exploited in the literature to manage arranged material circumstances. In this work, we consider a fractionalorder NS equation for an incompressible fluid flow of kinematic viscosity $v=\frac{\phi}{\rho}$ and density $\rho$. It is indicated as

$$
\left\{\begin{array}{l}
D_{\eta}^{\beta} V+(V . \nabla) V=\rho \nabla^{2} V-\frac{1}{\rho} \nabla g, \\
\nabla \cdot V=0, \\
V=0, \quad \text { on } \Omega \times(0, T) .
\end{array}\right.
$$

Here, $V=(\mathcal{U}, \mathcal{V}, \mathcal{W}), q$, and $\eta$ represent fluid vector, pressure, and time, respectively. $(\chi, \varphi, \mathcal{Z})$ represents the spatial components in $\Omega . \phi$ is the dynamic viscosity. $\rho$ is the density and the ratio of $\phi$.

(c) The Author(s) 2021. This article is licensed under a Creative Commons Attribution 4.0 International License, which permits use, sharing, adaptation, distribution and reproduction in any medium or format, as long as you give appropriate credit to the original author(s) and the source, provide a link to the Creative Commons licence, and indicate if changes were made. The images or other third party material in this article are included in the article's Creative Commons licence, unless indicated otherwise in a credit line to the material. If material is not included in the article's Creative Commons licence and your intended use is not permitted by statutory regulation or exceeds the permitted use, you will need to obtain permission directly from the copyright holder. To view a copy of this licence, visit http://creativecommons.org/licenses/by/4.0/. 
The above equations can also be defined as

$$
\begin{aligned}
& D_{\eta}^{\Upsilon}(\Phi)+\Phi \frac{\partial \Phi}{\partial \chi}+\Psi \frac{\partial \Phi}{\partial \varphi}+\Theta \frac{\partial \Phi}{\partial \mathcal{Z}}=\rho\left[\frac{\partial^{2} \Phi}{\partial \chi^{2}}+\frac{\partial^{2} \Phi}{\partial \varphi^{2}}+\frac{\partial^{2} \Phi}{\partial \mathcal{Z}^{2}}\right]-\frac{1}{\rho} \frac{\partial g}{\partial \chi}, \\
& D_{\eta}^{\Upsilon}(\Psi)+\Phi \frac{\partial \Psi}{\partial \chi}+\Psi \frac{\partial \Psi}{\partial \varphi}+\Theta \frac{\partial \Psi}{\partial \mathcal{Z}}=\rho\left[\frac{\partial^{2} \Psi}{\partial \chi^{2}}+\frac{\partial^{2} \Psi}{\partial \varphi^{2}}+\frac{\partial^{2} \Psi}{\partial \mathcal{Z}^{2}}\right]-\frac{1}{\rho} \frac{\partial g}{\partial \varphi}, \\
& D_{\eta}^{\Upsilon}(\Theta)+\Phi \frac{\partial \Theta}{\partial \chi}+\Psi \frac{\partial \Theta}{\partial \varphi}+\Theta \frac{\partial \Theta}{\partial \mathcal{Z}}=\rho\left[\frac{\partial^{2} \Theta}{\partial \chi^{2}}+\frac{\partial^{2} \Theta}{\partial \varphi^{2}}+\frac{\partial^{2} \Theta}{\partial \mathcal{Z}^{2}}\right]-\frac{1}{\rho} \frac{\partial g}{\partial \mathcal{Z}} .
\end{aligned}
$$

Mathematically, these equations are a problematic arrangement of nonlinear equations within sight of viscous flows [1].

Physical marvels of the incidental fields can be demonstrated properly using fractional partial differential equations (FPDEs). The hypothesis of partial analytic is outfitted with fabulous instruments to depict the dynamical conduct and memory-related qualities of logical frameworks and cycles from the last three decades. Different researchers have utilized FDEs in the displaying and investigation of logical phenomena in various fields of knowledge [2-11]. The hypothesis of fractional calculus has been generally used in different fields. It is becoming extremely quick in creating models because of its connection with memory and fractals plentiful in genuine physical frameworks. Fractional calculus demonstrating limits the mistake that emerges from the numbness of noteworthy genuine boundaries. It allows a more extraordinary level of opportunity in the model contrasted with an integral-order framework [12, 13]. FDEs are furnished with magnificent strategies to portray innate and memory attributes that are essentially disregarded by the whole number order framework. Likewise, they are also appropriate in demonstrating genuine frameworks and important in the examination of dynamical frameworks. FDEs are likewise suitable if there should be an occurrence of displaying frameworks with longer-go intuitiveness both in space and time. The soundness area increments in the event of a fractional-order framework when contrasted with its whole number order system. Fractional analytic likewise gives nonlocal operators and mathematical outcomes with high precision [14, 15]. Likewise, fractional-order frameworks, at last, meet the whole number order frameworks.

The nonlocal characteristic of the fractional operator is the most profitable element in this situation. The hypothesis of fractional analytic creates numerous speculations concerning non-neighborhood qualities, improved level of opportunity, most excessive use of data, and these attributes happen on account of fractional-order frameworks. The mathematical plans given by fractional analytic begin the more profound comprehension of complex frameworks and diminish the computational work concerning the solutions strategy [16-24]. Precise expository solutions are not found virtually on account of FDEs. Hence, over the most recent twenty years, numerous iterative plans, for example, homotopy perturbation method, Adomian decomposition method, variational iteration method, homotopy perturbation transform strategy, residual power series strategy, and so forth, have been created to obtain the solutions of a few classes of FDEs [25-36].

A Chinese mathematician has created the variational iteration method (VIM) He [37]. VIM is modified with the Laplace transform; the modified method is known as the variational iteration transform method (VITM). After the original work of He, a different adjustment of VIM has been utilized to take care of different nonlinear issues, for example, 
dissemination and wave equations [38-45]. Recently, several mathematicians have applied various strategies for the solutions of fractional NSEs. Interested readers can see [46-51] and the references therein.

In this paper, the variational iteration transform technique is implemented to analyze the solution of fractional-order multi-dimensional Navier-Stokes equations. Caputo operator describes the fractional-order derivatives. The solution of certain illustrative problems is provided to prove the feasibility of the proposed methodology. The results of the proposed method are shown and analyzed with the help of figures and tables. The current approach has lower computing costs and higher convergence rates. The proposed method is therefore constructive to solve other fractional-order PDEs.

The outline of this article is as follows. In Sect. 2, the basic definitions of Laplace transform and fractional calculus are discussed. In Sect. 3, the variational iteration transform method is discussed. In Sect. 4, three test examples of fractional-order Navier-Stokes equation are given to elucidate the suggested schemes. In Sect. 5, conclusions of the work are drawn.

\section{Basic definitions}

Definition 2.1 The fractional-order derivative of $g(\chi)$ in the Caputo sense is given as

$$
\begin{gathered}
D^{\Upsilon} g(\chi)=\frac{1}{\Gamma(k-\Upsilon)} \int_{0}^{\chi}(\chi-\tau)^{k-\Upsilon-1} g^{(k)}(\tau) d \tau, \\
\text { for } k-1<\Upsilon<k, k \in N, \chi>0, g \in C_{-1}^{k} .
\end{gathered}
$$

Definition 2.2 The Laplace transformation of $g(\chi), \tau>0$ is expressed as

$$
F(s)=L[g(\tau)]=\int_{0}^{\infty} e^{-s \tau} g(\tau) d \tau .
$$

Definition 2.3 The Laplace transformation $L[g(\tau)]$ of the Caputo derivative is given as

$$
L\left[D^{\Upsilon} g(\tau)\right]=s^{\Upsilon} F(s)-\sum_{m=0}^{k-1} s^{\Upsilon-1-k} g^{m}(0), \quad k-1<\Upsilon<k .
$$

Definition 2.4 The Mittag-Leffler function $E_{\Upsilon}(z)$ with $\Upsilon>0$ is given by

$$
E_{\Upsilon}(z)=\sum_{k=0}^{\infty} \frac{z^{k}}{\Gamma(\Upsilon k+1)}, \quad \Upsilon>0, z \in C .
$$

\section{The procedure of VITM}

This section describes the VITM solution of fractional PDEs [52, 53].

$$
\begin{aligned}
& D_{\eta}^{\Upsilon} \Phi(\chi, \eta)+\mathcal{H}_{1}(\Phi, \Psi)+\mathcal{M}_{1}(\Phi, \Psi)-q_{1}(\chi, \eta)=0, \\
& D_{\eta}^{\Upsilon} \Psi(\chi, \eta)+\mathcal{H}_{2}(\Phi, \Psi)+\mathcal{M}_{2}(\Phi, \Psi)-q_{2}(\chi, \eta)=0, \quad m-1<\Upsilon \leq m,
\end{aligned}
$$

with the initial conditions

$$
\Phi(\chi, 0)=g_{1}(\chi), \quad \Psi(\chi, 0)=g_{2}(\chi)
$$


where is $D_{\eta}^{\Upsilon}=\frac{\partial^{\Upsilon}}{\partial \eta^{\Upsilon}}$ the Caputo fractional derivative of order $\Upsilon, \mathcal{H}_{1}, \mathcal{H}_{2}$ and $\mathcal{M}_{1}, \mathcal{M}_{2}$ are linear and nonlinear functions, respectively, and $q_{1}, q_{2}$ are source operators.

The Laplace transformation is applied to Eq. (1),

$$
\begin{aligned}
& L\left[D_{\eta}^{\Upsilon} \Phi(\chi, \eta)\right]+L\left[\mathcal{H}_{1}(\Phi, \Psi)+\mathcal{M}_{1}(\Phi, \Psi)-q_{1}(\chi, \eta)\right]=0, \\
& L\left[D_{\eta}^{\Upsilon} \Psi(\chi, \eta)\right]+L\left[\mathcal{H}_{2}(\Phi, \Psi)+\mathcal{M}_{2}(\Phi, \Psi)-q_{2}(\chi, \eta)\right]=0 .
\end{aligned}
$$

Applying the differentiation property, we have

$$
\begin{aligned}
& L[\Phi(\chi, \eta)]-\left.\sum_{k=0}^{m-1} s^{\Upsilon-k-1} \frac{\partial^{k} \Phi(\chi, \eta)}{\partial^{k} \eta}\right|_{\eta=0}=-L\left[\mathcal{H}_{1}(\Phi, \Psi)+\mathcal{M}_{1}(\Phi, \Psi)-q_{1}(\chi, \eta)\right], \\
& L[\Psi(\chi, \eta)]-\left.\sum_{k=0}^{m-1} s^{\Upsilon-k-1} \frac{\partial^{k} \Psi(\chi, \eta)}{\partial^{k} \eta}\right|_{\eta=0}=-L\left[\mathcal{H}_{2}(\Phi, \Psi)+\mathcal{M}_{2}(\Phi, \Psi)-q_{2}(\chi, \eta)\right] .
\end{aligned}
$$

Using the iterative technique, we get

$$
\begin{aligned}
L\left[\Phi_{m+1}(\chi, \eta)\right]= & L\left[\Phi_{m}(\chi, \eta)\right] \\
& +\lambda(s)\left[s^{\Upsilon} \Phi_{m}(\chi, \eta)-\left.\sum_{k=0}^{m-1} s^{\Upsilon-k-1} \frac{\partial^{k} \Phi(\chi, \eta)}{\partial^{k} \eta}\right|_{\eta=0}-L\left[q_{1}(\chi, \eta)\right]\right. \\
& \left.-L\left\{\mathcal{H}_{1}(\Phi, \Psi)+\mathcal{M}_{1}(\Phi, \Psi)\right\}\right], \\
L\left[\Psi_{m+1}(\chi, \eta)\right]= & L\left[\Psi_{m}(\chi, \eta)\right] \\
& +\lambda(s)\left[s^{\Upsilon} \Psi_{m}(\chi, \eta)-\left.\sum_{k=0}^{m-1} s^{\Upsilon-k-1} \frac{\partial^{k} \Psi(\chi, \eta)}{\partial^{k} \eta}\right|_{\eta=0}-L\left[q_{2}(\chi, \eta)\right]\right. \\
& \left.-L\left\{\mathcal{H}_{2}(\Phi, \Psi)+\mathcal{M}_{2}(\Phi, \Psi)\right\}\right] .
\end{aligned}
$$

A Lagrange multiplier as

$$
\lambda(s)=-\frac{1}{s^{\Upsilon}},
$$

the inverse Laplace transformation $L^{-1}$, the iteration method Eq. (5) can be given as follows:

$$
\begin{aligned}
\Phi_{m+1}(\chi, \eta)= & \Phi_{m}(\chi, \eta)-L^{-1}\left[\frac { 1 } { s ^ { \Upsilon } } \left[\left.\sum_{k=0}^{m-1} s^{\Upsilon-k-1} \frac{\partial^{k} \Phi(\chi, \eta)}{\partial^{k} \eta}\right|_{\eta=0}\right.\right. \\
& \left.\left.-L\left[q_{1}(\chi, \eta)\right]-L\left\{\mathcal{H}_{1}(\Phi, \Psi)+\mathcal{M}_{1}(\Phi, \Psi)\right\}\right]\right],
\end{aligned}
$$




$$
\begin{aligned}
\Psi_{m+1}(\chi, \eta)= & \Psi_{m}(\chi, \eta)-L^{-1}\left[\frac { 1 } { s ^ { \Upsilon } } \left[\left.\sum_{k=0}^{m-1} s^{\Upsilon-k-1} \frac{\partial^{k} \Psi(\chi, \eta)}{\partial^{k} \eta}\right|_{\eta=0}\right.\right. \\
& \left.\left.-L\left[q_{2}(\chi, \eta)\right]-L\left\{\mathcal{H}_{2}(\Phi, \Psi)+\mathcal{M}_{2}(\Phi, \Psi)\right\}\right]\right]
\end{aligned}
$$

The initial iteration can be found as follows:

$$
\begin{aligned}
& \Phi_{0}(\chi, \eta)=L^{-1}\left[\frac{1}{s^{\Upsilon}}\left\{\left.\sum_{k=0}^{m-1} s^{\Upsilon-k-1} \frac{\partial^{k} \Phi(\chi, \eta)}{\partial^{k} \eta}\right|_{\eta=0}\right\}\right], \\
& \Psi_{0}(\chi, \eta)=L^{-1}\left[\frac{1}{s^{\Upsilon}}\left\{\left.\sum_{k=0}^{m-1} s^{\Upsilon-k-1} \frac{\partial^{k} \Psi(\chi, \eta)}{\partial^{k} \eta}\right|_{\eta=0}\right\}\right] .
\end{aligned}
$$

The conveniences of this technique were shown in $[52,53]$.

\section{Numerical examples}

Example 1 Consider the time fractional-order $(1+1)$ dimensional Navier-Stokes equation

$$
\begin{aligned}
& D_{\eta}^{\Upsilon}(\Phi)+\Phi \frac{\partial \Phi}{\partial \chi}+\Psi \frac{\partial \Phi}{\partial \varphi}=\rho\left[\frac{\partial^{2} \Phi}{\partial \chi^{2}}+\frac{\partial^{2} \Phi}{\partial \varphi^{2}}\right]+q, \\
& D_{\eta}^{\Upsilon}(\Psi)+\Phi \frac{\partial \Psi}{\partial \chi}+\Psi \frac{\partial \Psi}{\partial \varphi}=\rho\left[\frac{\partial^{2} \Psi}{\partial \chi^{2}}+\frac{\partial^{2} \Psi}{\partial \varphi^{2}}\right]-q,
\end{aligned}
$$

with the initial conditions

$$
\left\{\begin{array}{l}
\Phi(\chi, \varphi, 0)=-\sin (\chi+\varphi), \\
\Psi(\chi, \varphi, 0)=\sin (\chi+\varphi) .
\end{array}\right.
$$

Using the iterative method according to equation (7) in equation (9), we get

$$
\begin{aligned}
\Phi_{m+1}(\chi, \varphi, \eta)= & \Phi_{m}(\chi, \varphi, \eta)-L^{-1}\left[\frac { 1 } { s ^ { \Upsilon } } L \left\{s \Upsilon \frac{\partial \Phi_{m}}{\partial \eta}\right.\right. \\
& \left.\left.+\Phi_{m} \frac{\partial \Phi_{m}}{\partial \chi}+\Psi_{m} \frac{\partial \Phi_{m}}{\partial \varphi}-\rho\left(\frac{\partial^{2} \Phi_{m}}{\partial \chi^{2}}+\frac{\partial^{2} \Phi_{m}}{\partial \varphi^{2}}\right)-q\right\}\right], \\
\Psi_{m+1}(\chi, \varphi, \eta)= & \Psi_{m}(\chi, \varphi, \eta)-L^{-1}\left[\frac { 1 } { s ^ { \Upsilon } } L \left\{s \frac{\partial \Psi_{m}}{\partial \eta}\right.\right. \\
& \left.\left.+\Phi_{m} \frac{\partial \Psi_{m}}{\partial \chi}+\Psi_{m} \frac{\partial \Psi_{m}}{\partial \varphi}-\rho\left(\frac{\partial^{2} \Psi_{m}}{\partial \chi^{2}}+\frac{\partial^{2} \Psi_{m}}{\partial \varphi^{2}}\right)+q\right\}\right],
\end{aligned}
$$

where

$$
\Phi_{0}(\chi, \varphi, \eta)=-\sin (\chi+\varphi), \quad \Psi_{0}(\chi, \varphi, \eta)=\sin (\chi+\varphi) .
$$


For $m=0,1,2, \ldots$,

$$
\begin{aligned}
& \Phi_{1}(\chi, \varphi, \eta)=\Phi_{0}(\chi, \varphi, \eta) \\
& -L^{-1}\left[\frac{1}{s^{\Upsilon}} L\left\{s^{\Upsilon} \frac{\partial \Phi_{0}}{\partial \eta}+\Phi_{0} \frac{\partial \Phi_{0}}{\partial \chi}+\Psi_{0} \frac{\partial \Phi_{0}}{\partial \varphi}-\rho\left(\frac{\partial^{2} \Phi_{0}}{\partial \chi^{2}}+\frac{\partial^{2} \Phi_{0}}{\partial \varphi^{2}}\right)-q\right\}\right], \\
& \Psi_{1}(\chi, \varphi, \eta)=\Psi_{0}(\chi, \varphi, \eta) \\
& -L^{-1}\left[\frac{1}{s^{\Upsilon}} L\left\{s \frac{\partial \Psi_{0}}{\partial \eta}+\Phi_{0} \frac{\partial \Psi_{0}}{\partial \chi}+\Psi_{0} \frac{\partial \Psi_{0}}{\partial \varphi}-\rho\left(\frac{\partial^{2} \Psi_{0}}{\partial \chi^{2}}+\frac{\partial^{2} \Psi_{0}}{\partial \varphi^{2}}\right)+q\right\}\right], \\
& \Phi_{1}(\chi, \varphi, \eta)=-\sin (\chi+\varphi)+\sin (\chi+\varphi) \frac{2 \rho \eta^{\Upsilon}}{\Gamma(\Upsilon+1)}+\frac{q \eta^{\Upsilon}}{\Gamma(\Upsilon+1)}, \\
& \Psi_{1}(\chi, \varphi, \eta)=\sin (\chi+\varphi)-\sin (\chi+\varphi) \frac{2 \rho \eta^{\Upsilon}}{\Gamma(\Upsilon+1)}-\frac{q \eta^{\Upsilon}}{\Gamma(\Upsilon+1)}, \\
& \Phi_{2}(\chi, \varphi, \eta)=\Phi_{1}(\chi, \varphi, \eta) \\
& -L^{-1}\left[\frac{1}{s^{\Upsilon}} L\left\{s^{\Upsilon} \frac{\partial \Phi_{1}}{\partial \eta}+\Phi_{1} \frac{\partial \Phi_{1}}{\partial \chi}+\Psi_{1} \frac{\partial \Phi_{1}}{\partial \varphi}-\rho\left(\frac{\partial^{2} \Phi_{1}}{\partial \chi^{2}}+\frac{\partial^{2} \Phi_{1}}{\partial \varphi^{2}}\right)-q\right\}\right], \\
& \Psi_{2}(\chi, \varphi, \eta)=\Psi_{1}(\chi, \varphi, \eta) \\
& -L^{-1}\left[\frac{1}{s^{\Upsilon}} L\left\{s^{\Upsilon} \frac{\partial \Psi_{1}}{\partial \eta}+\Phi_{1} \frac{\partial \Psi_{1}}{\partial \chi}+\Psi_{1} \frac{\partial \Psi_{1}}{\partial \varphi}-\rho\left(\frac{\partial^{2} \Psi_{1}}{\partial \chi^{2}}+\frac{\partial^{2} \Psi_{1}}{\partial \varphi^{2}}\right)+q\right\}\right], \\
& \Phi_{2}(\chi, \varphi, \eta)=-\sin (\chi+\varphi)+\sin (\chi+\varphi) \frac{2 \rho \eta^{\Upsilon}}{\Gamma(\Upsilon+1)}+\frac{q \eta^{\Upsilon}}{\Gamma(\Upsilon+1)}-\sin (\chi+\varphi) \frac{(2 \rho)^{2} \eta^{2 \Upsilon}}{\Gamma(2 \Upsilon+1)}, \\
& \Psi_{2}(\chi, \varphi, \eta)=\sin (\chi+\varphi)-\sin (\chi+\varphi) \frac{2 \rho \eta^{\Upsilon}}{\Gamma(\Upsilon+1)}-\frac{q \eta^{\Upsilon}}{\Gamma(\Upsilon+1)}+\sin (\chi+\varphi) \frac{(2 \rho)^{2} \eta^{2 \Upsilon}}{\Gamma(2 \Upsilon+1)}, \\
& \Phi_{3}(\chi, \varphi, \eta)=\Phi_{2}(\chi, \varphi, \eta) \\
& -L^{-1}\left[\frac{1}{s^{\Upsilon}} L\left\{s^{\Upsilon} \frac{\partial \Phi_{2}}{\partial \eta}+\Phi_{2} \frac{\partial \Phi_{2}}{\partial \chi}+\Psi_{2} \frac{\partial \Phi_{2}}{\partial \varphi}-\rho\left(\frac{\partial^{2} \Phi_{2}}{\partial \chi^{2}}+\frac{\partial^{2} \Phi_{2}}{\partial \varphi^{2}}\right)-q\right\}\right], \\
& \Psi_{3}(\chi, \varphi, \eta)=\Psi_{2}(\chi, \varphi, \eta) \\
& -L^{-1}\left[\frac{1}{s^{\Upsilon}} L\left\{s^{\Upsilon} \frac{\partial \Psi_{2}}{\partial \eta}+\Phi_{2} \frac{\partial \Psi_{2}}{\partial \chi}+\Psi_{2} \frac{\partial \Psi_{2}}{\partial \varphi}-\rho\left(\frac{\partial^{2} \Psi_{2}}{\partial \chi^{2}}+\frac{\partial^{2} \Psi_{2}}{\partial \varphi^{2}}\right)+q\right\}\right], \\
& \Phi_{3}(\chi, \varphi, \eta)=-\sin (\chi+\varphi)+\sin (\chi+\varphi) \frac{2 \rho \eta^{\Upsilon}}{\Gamma(\Upsilon+1)}+\frac{q \eta^{\Upsilon}}{\Gamma(\Upsilon+1)} \\
& -\sin (\chi+\varphi) \frac{(2 \rho)^{2} \eta^{2 \Upsilon}}{\Gamma(2 \Upsilon+1)}+\sin (\chi+\varphi) \frac{(2 \rho)^{3} \eta^{3 \Upsilon}}{\Gamma(3 \Upsilon+1)}, \\
& \Psi_{3}(\chi, \varphi, \eta)=\sin (\chi+\varphi)-\sin (\chi+\varphi) \frac{2 \rho \eta^{\Upsilon}}{\Gamma(\Upsilon+1)}-\frac{q \eta^{\Upsilon}}{\Gamma(\Upsilon+1)} \\
& +\sin (\chi+\varphi) \frac{(2 \rho)^{2} \eta^{2 \Upsilon}}{\Gamma(2 \Upsilon+1)}-\sin (\chi+\varphi) \frac{(2 \rho)^{3} \eta^{3 \Upsilon}}{\Gamma(3 \Upsilon+1)}, \\
& \Phi(\chi, \varphi, \eta)=\sum_{m=0}^{\infty} \Phi_{m}(\chi, \varphi)=-\sin (\chi+\varphi) \sum_{m=0}^{\infty} \frac{(-2 \rho)^{m} \eta^{m \Upsilon}}{\Gamma(m \Upsilon+1)}+\frac{q \eta^{\Upsilon}}{\Gamma(\Upsilon+1)}, \\
& \Psi(\chi, \varphi, \eta)=\sum_{m=0}^{\infty} \Phi_{m}(\chi, \varphi)=\sin (\chi+\varphi) \sum_{m=0}^{\infty} \frac{(-2 \rho)^{m} \eta^{m}}{\Gamma(m \Upsilon+1)}-\frac{q \eta^{\Upsilon}}{\Gamma(\Upsilon+1)}
\end{aligned}
$$


The exact solution of equation (9) at $\Upsilon=1$ and $q=0$,

$$
\begin{aligned}
& \Phi(\chi, \varphi, \eta)=-e^{-2 \rho \eta} \sin (\chi+\varphi), \\
& \Psi(\chi, \varphi, \eta)=e^{-2 \rho \eta} \sin (\chi+\varphi) .
\end{aligned}
$$

Figures 1 and 4 show the behavior of solutions of the exact and analytical results using the initial conditions given in equation (10). In Fig. 1 the exact and analytical solutions of $\Phi$ at $\Upsilon=1$ show close contact with each other. In Figs. 2 and 3 for different values of $\Upsilon=0.8,0.6$, and 0.4 for $\Phi$. In Fig. 4, we show the exact and analytical solution of $\Psi$ at $\Upsilon=1$. In Figs. 5 and 6 for different values of $\Upsilon=0.8,0.6$, and 0.4 for $\Psi$. The fractional results are investigated to be convergent to an integer-order result of each problem.

Example 2 Consider the time fractional-order $(1+1)$ dimensional Navier-Stokes equation

$$
\begin{aligned}
& D_{\eta}^{\Upsilon}(\Phi)+\Phi \frac{\partial \Phi}{\partial \chi}+\Psi \frac{\partial \Phi}{\partial \varphi}=\rho\left[\frac{\partial^{2} \Phi}{\partial \chi^{2}}+\frac{\partial^{2} \Phi}{\partial \varphi^{2}}\right]+q, \\
& D_{\eta}^{\Upsilon}(\Psi)+\Phi \frac{\partial \Psi}{\partial \chi}+\Psi \frac{\partial \Psi}{\partial \varphi}=\rho\left[\frac{\partial^{2} \Psi}{\partial \chi^{2}}+\frac{\partial^{2} \Psi}{\partial \varphi^{2}}\right]-q,
\end{aligned}
$$
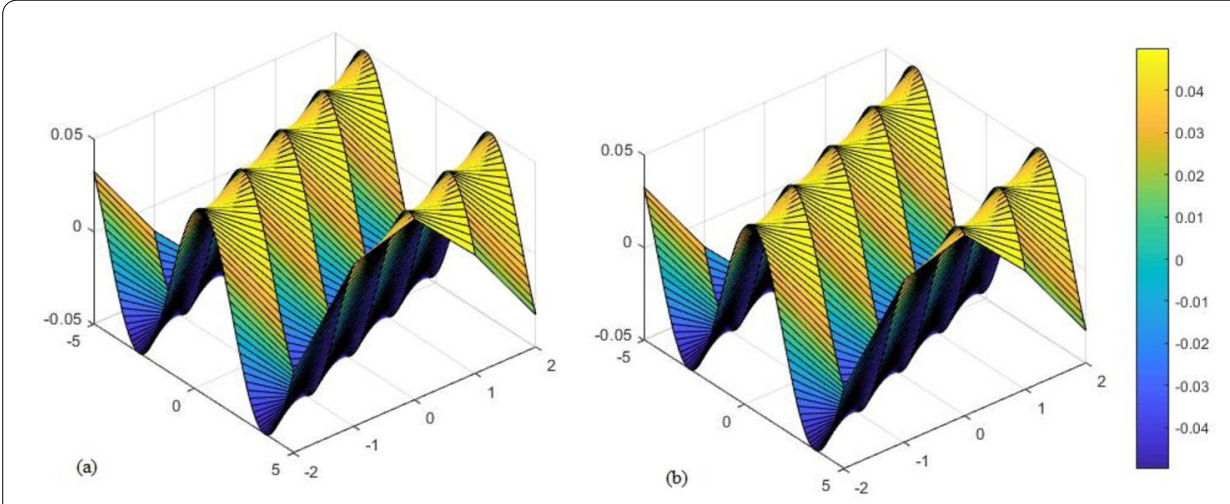

Figure 1 The graph of exact (a) and approximate (b) solution of $\Phi(\chi, \varphi, \eta)$ at $\Upsilon=1$ of Example 1
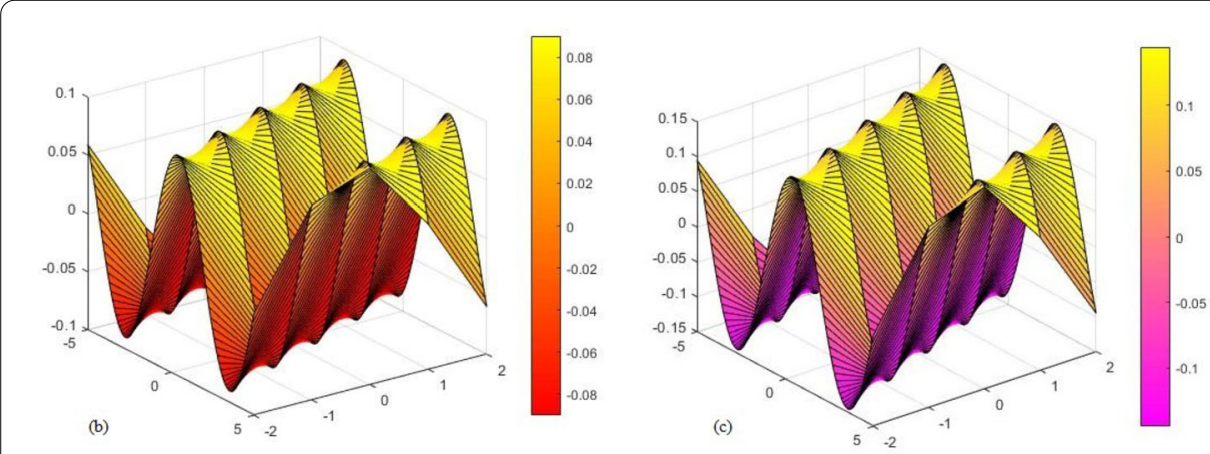

Figure 2 The graph of different approximate solution of $\Phi(\chi, \varphi, \eta)$ at $\Upsilon=(\mathbf{c}) 0.8$ and (d) 0.6 of Example 1 


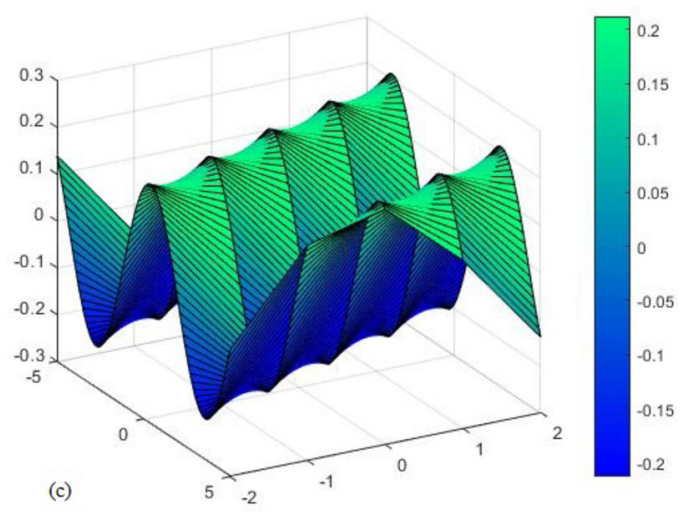

Figure 3 The graph of approximate solution (e) of $\Phi(\chi, \varphi, \eta)$ at $\Upsilon=0.4$ of Example 1
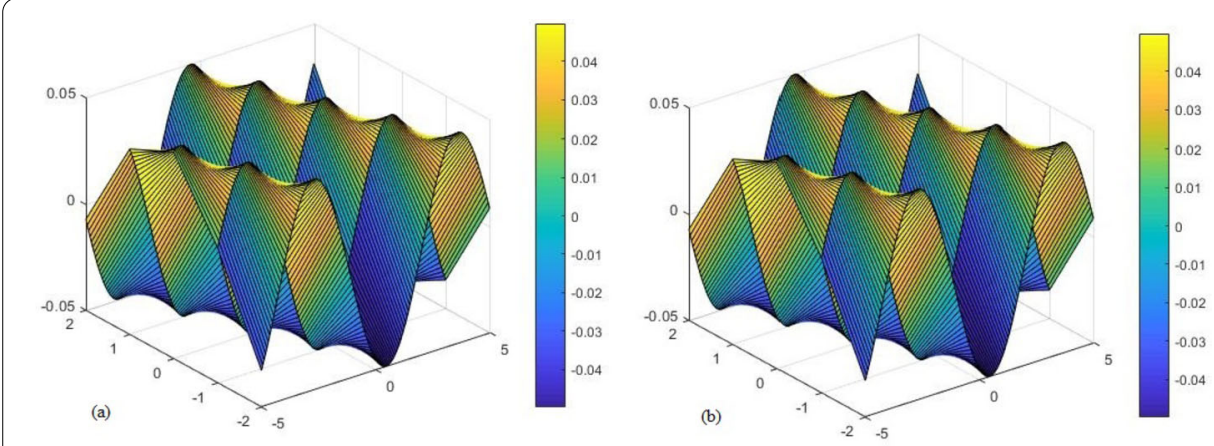

Figure 4 The graph of exact (a) and approximate (b) solution of $\Psi(\chi, \varphi, \eta)$ at $\Upsilon=1$ of Example 1
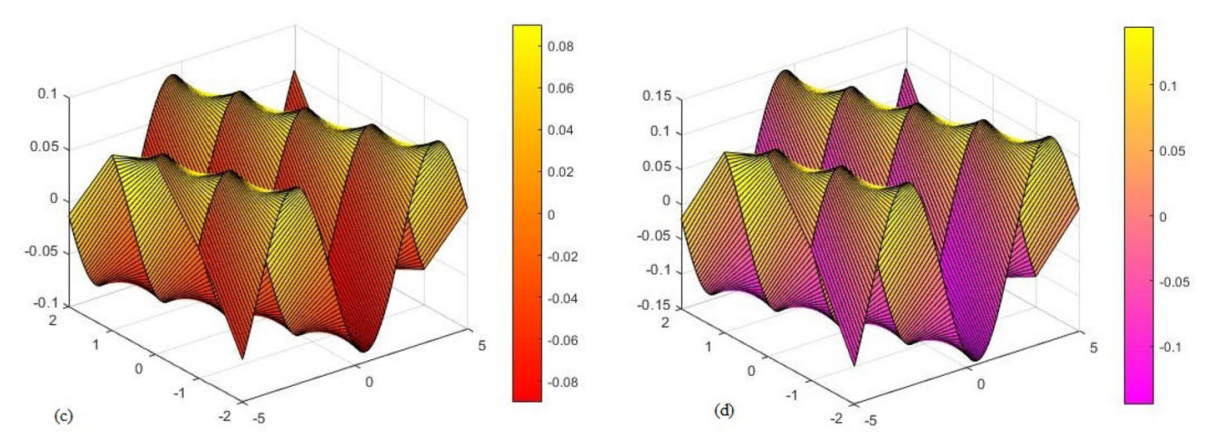

Figure 5 The graph of different approximate solution of $\Psi(\chi, \varphi, \eta)$ at $\Upsilon=(\mathbf{c}) 0.8$ and $(\mathbf{d}) 0.6$ of Example 1

with the initial conditions

$$
\left\{\begin{array}{l}
\Phi(\chi, \varphi, 0)=-e^{\chi+\varphi} \\
\Psi(\chi, \varphi, 0)=e^{\chi+\varphi}
\end{array}\right.
$$




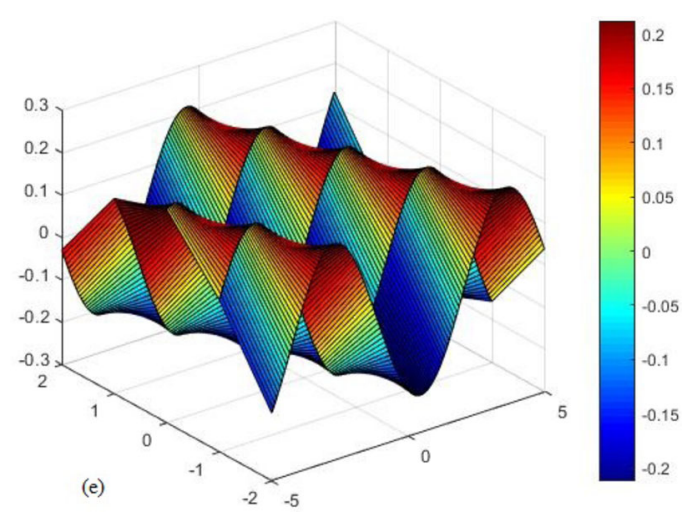

Figure 6 The graph of approximate solution (e) of $\Psi(\chi, \varphi, \eta)$ at $\Upsilon=0.4$ of Example 1

Using the iterative method according to equation (7) in equation (15), we get

$$
\begin{aligned}
\Phi_{m+1}(\chi, \varphi, \eta)= & \Phi_{m}(\chi, \varphi, \eta)-L^{-1}\left[\frac { 1 } { s ^ { \Upsilon } } L \left\{s \Upsilon \frac{\partial \Phi_{m}}{\partial \eta}\right.\right. \\
& \left.\left.+\Phi_{m} \frac{\partial \Phi_{m}}{\partial \chi}+\Psi_{m} \frac{\partial \Phi_{m}}{\partial \varphi}-\rho\left(\frac{\partial^{2} \Phi_{m}}{\partial \chi^{2}}+\frac{\partial^{2} \Phi_{m}}{\partial \varphi^{2}}\right)-q\right\}\right], \\
\Psi_{m+1}(\chi, \varphi, \eta)= & \Psi_{m}(\chi, \varphi, \eta)-L^{-1}\left[\frac { 1 } { s ^ { \Upsilon } } L \left\{s \frac{\partial \Psi_{m}}{\partial \eta}\right.\right. \\
& \left.\left.+\Phi_{m} \frac{\partial \Psi_{m}}{\partial \chi}+\Psi_{m} \frac{\partial \Psi_{m}}{\partial \varphi}-\rho\left(\frac{\partial^{2} \Psi_{m}}{\partial \chi^{2}}+\frac{\partial^{2} \Psi_{m}}{\partial \varphi^{2}}\right)+q\right\}\right],
\end{aligned}
$$

where

$$
\Phi_{0}(\chi, \varphi, \eta)=-e^{\chi+\varphi}, \quad \Psi_{0}(\chi, \varphi, \eta)=e^{\chi+\varphi} .
$$

For $m=0,1,2, \ldots$,

$$
\begin{aligned}
\Phi_{1}(\chi, \varphi, \eta)= & \Phi_{0}(\chi, \varphi, \eta) \\
& -L^{-1}\left[\frac{1}{s^{\Upsilon}} L\left\{s \Upsilon \frac{\partial \Phi_{0}}{\partial \eta}+\Phi_{0} \frac{\partial \Phi_{0}}{\partial \chi}+\Psi_{0} \frac{\partial \Phi_{0}}{\partial \varphi}-\rho\left(\frac{\partial^{2} \Phi_{0}}{\partial \chi^{2}}+\frac{\partial^{2} \Phi_{0}}{\partial \varphi^{2}}\right)-q\right\}\right], \\
\Psi_{1}(\chi, \varphi, \eta)= & \Psi_{0}(\chi, \varphi, \eta) \\
& -L^{-1}\left[\frac{1}{s^{\Upsilon}} L\left\{s^{\Upsilon} \frac{\partial \Psi_{0}}{\partial \eta}+\Phi_{0} \frac{\partial \Psi_{0}}{\partial \chi}+\Psi_{0} \frac{\partial \Psi_{0}}{\partial \varphi}-\rho\left(\frac{\partial^{2} \Psi_{0}}{\partial \chi^{2}}+\frac{\partial^{2} \Psi_{0}}{\partial \varphi^{2}}\right)+q\right\}\right], \\
\Phi_{1}(\chi, \varphi, \eta)= & -e^{\chi+\varphi}+e^{\chi+\varphi} \frac{2 \rho \eta}{\Gamma(\Upsilon+1)}+\frac{q \eta^{\Upsilon}}{\Gamma(\Upsilon+1)}, \\
\Psi_{1}(\chi, \varphi, \eta)= & e^{\chi+\varphi}-e^{\chi+\varphi} \frac{2 \rho \eta^{\Upsilon}}{\Gamma(\Upsilon+1)}-\frac{q \eta^{\Upsilon}}{\Gamma(\Upsilon+1)}, \\
\Phi_{2}(\chi, \varphi, \eta)= & \Phi_{1}(\chi, \varphi, \eta) \\
& -L^{-1}\left[\frac{1}{s^{\Upsilon}} L\left\{s^{\Upsilon} \frac{\partial \Phi_{1}}{\partial \eta}+\Phi_{1} \frac{\partial \Phi_{1}}{\partial \chi}+\Psi_{1} \frac{\partial \Phi_{1}}{\partial \varphi}-\rho\left(\frac{\partial^{2} \Phi_{1}}{\partial \chi^{2}}+\frac{\partial^{2} \Phi_{1}}{\partial \varphi^{2}}\right)-q\right\}\right],
\end{aligned}
$$




$$
\begin{aligned}
& \Psi_{2}(\chi, \varphi, \eta)=\Psi_{1}(\chi, \varphi, \eta) \\
& -L^{-1}\left[\frac{1}{s^{\Upsilon}} L\left\{s^{\Upsilon} \frac{\partial \Psi_{1}}{\partial \eta}+\Phi_{1} \frac{\partial \Psi_{1}}{\partial \chi}+\Psi_{1} \frac{\partial \Psi_{1}}{\partial \varphi}-\rho\left(\frac{\partial^{2} \Psi_{1}}{\partial \chi^{2}}+\frac{\partial^{2} \Psi_{1}}{\partial \varphi^{2}}\right)+q\right\}\right], \\
& \Phi_{2}(\chi, \varphi, \eta)=-e^{\chi+\varphi}+e^{\chi+\varphi} \frac{2 \rho \eta^{\Upsilon}}{\Gamma(\Upsilon+1)}+\frac{q \eta^{\Upsilon}}{\Gamma(\Upsilon+1)}-e^{\chi+\varphi} \frac{(2 \rho)^{2} \eta^{2 \Upsilon}}{\Gamma(2 \Upsilon+1)}, \\
& \Psi_{2}(\chi, \varphi, \eta)=e^{\chi+\varphi}-e^{\chi+\varphi} \frac{2 \rho \eta^{\Upsilon}}{\Gamma(\Upsilon+1)}-\frac{q \eta^{\Upsilon}}{\Gamma(\Upsilon+1)}+e^{\chi+\varphi} \frac{(2 \rho)^{2} \eta^{2 \Upsilon}}{\Gamma(2 \Upsilon+1)}, \\
& \Phi_{3}(\chi, \varphi, \eta)=\Phi_{2}(\chi, \varphi, \eta) \\
& -L^{-1}\left[\frac{1}{s^{\Upsilon}} L\left\{s^{\Upsilon} \frac{\partial \Phi_{2}}{\partial \eta}+\Phi_{2} \frac{\partial \Phi_{2}}{\partial \chi}+\Psi_{2} \frac{\partial \Phi_{2}}{\partial \varphi}-\rho\left(\frac{\partial^{2} \Phi_{2}}{\partial \chi^{2}}+\frac{\partial^{2} \Phi_{2}}{\partial \varphi^{2}}\right)-q\right\}\right], \\
& \Psi_{3}(\chi, \varphi, \eta)=\Psi_{2}(\chi, \varphi, \eta) \\
& -L^{-1}\left[\frac{1}{s^{\Upsilon}} L\left\{s^{\Upsilon} \frac{\partial \Psi_{2}}{\partial \eta}+\Phi_{2} \frac{\partial \Psi_{2}}{\partial \chi}+\Psi_{2} \frac{\partial \Psi_{2}}{\partial \varphi}-\rho\left(\frac{\partial^{2} \Psi_{2}}{\partial \chi^{2}}+\frac{\partial^{2} \Psi_{2}}{\partial \varphi^{2}}\right)+q\right\}\right], \\
& \Phi_{3}(\chi, \varphi, \eta)=-e^{\chi+\varphi}+e^{\chi+\varphi} \frac{2 \rho \eta^{\Upsilon}}{\Gamma(\Upsilon+1)}+\frac{q \eta^{\Upsilon}}{\Gamma(\Upsilon+1)}-e^{\chi+\varphi} \frac{(2 \rho)^{2} \eta^{2 \Upsilon}}{\Gamma(2 \Upsilon+1)}+e^{\chi+\varphi} \frac{(2 \rho)^{3} \eta^{3 \Upsilon}}{\Gamma(3 \Upsilon+1)} \text {, } \\
& \Psi_{3}(\chi, \varphi, \eta)=e^{\chi+\varphi}-e^{\chi+\varphi} \frac{2 \rho \eta^{\Upsilon}}{\Gamma(\Upsilon+1)}-\frac{q \eta^{\Upsilon}}{\Gamma(\Upsilon+1)}+e^{\chi+\varphi} \frac{(2 \rho)^{2} \eta^{2 \Upsilon}}{\Gamma(2 \Upsilon+1)}-e^{\chi+\varphi} \frac{(2 \rho)^{3} \eta^{3 \Upsilon}}{\Gamma(3 \Upsilon+1)} \text {, } \\
& \Phi(\chi, \varphi, \eta)=\sum_{m=0}^{\infty} \Phi_{m}(\chi, \varphi)=-e^{\chi+\varphi} \sum_{m=0}^{\infty} \frac{(-2 \rho)^{m} \eta^{m \Upsilon}}{\Gamma(m \Upsilon+1)}+\frac{q \eta^{\Upsilon}}{\Gamma(\Upsilon+1)} \\
& \Psi(\chi, \varphi, \eta)=\sum_{m=0}^{\infty} \Phi_{m}(\chi, \varphi)=e^{\chi+\varphi} \sum_{m=0}^{\infty} \frac{(-2 \rho)^{m} \eta^{m \Upsilon}}{\Gamma(m \Upsilon+1)}-\frac{q \eta^{\Upsilon}}{\Gamma(\Upsilon+1)} .
\end{aligned}
$$

The exact result of equation (15) at $\Upsilon=1$ and $q=0$,

$$
\begin{aligned}
& \Phi(\chi, \varphi, \eta)=-e^{\chi+\varphi+2 \rho \eta} \\
& \Psi(\chi, \varphi, \eta)=e^{\chi+\varphi+2 \rho \eta} .
\end{aligned}
$$

Figures 7 and 10 show the behavior of solutions of the exact and analytical results using the initial conditions given in equation (16). In Fig. 7, the exact and analytical solutions of $\Phi$ at $\Upsilon=1$ show close contact with each other. In Figs. 7 and 9 for different values of

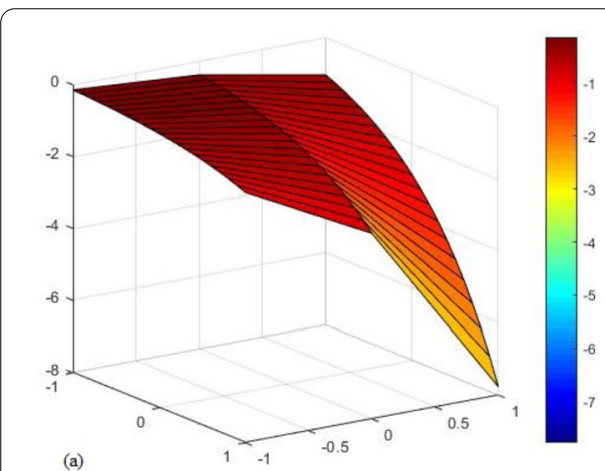

(a)

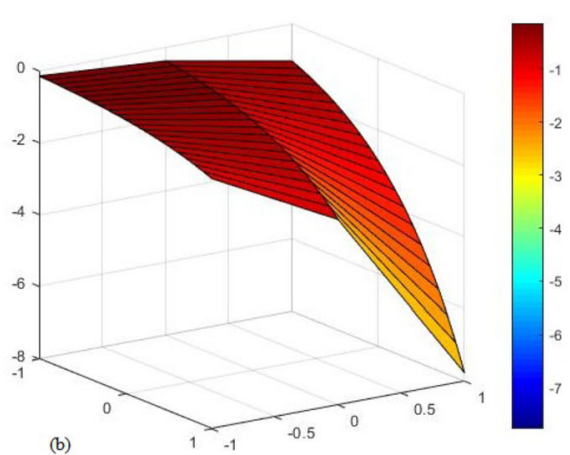

Figure 7 The graph of exact (a) and approximate (b) solution of $\Phi(\chi, \varphi, \eta)$ at $\Upsilon=1$ of Example 2 

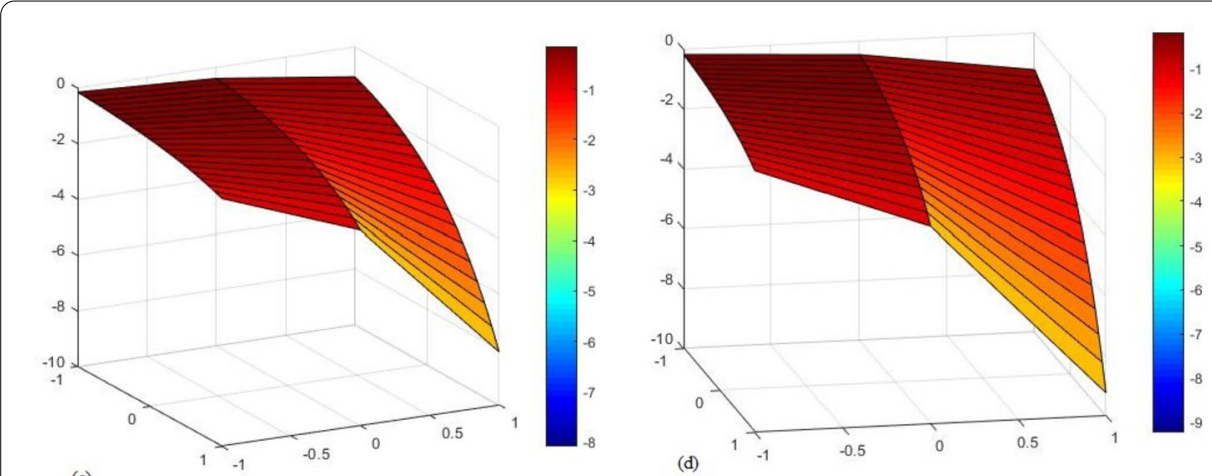

Figure 8 The graph of different approximate solution of $\Phi(\chi, \varphi, \eta)$ at $\Upsilon=(\mathbf{c}) 0.8$ and (d) 0.6 of Example 2

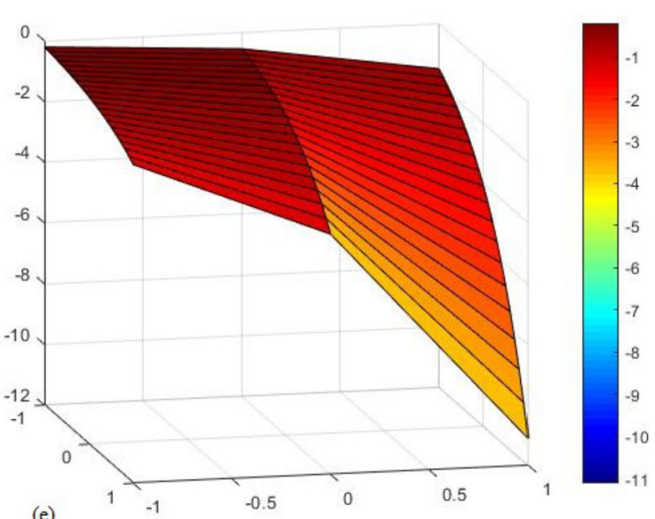

Figure 9 The graph of approximate (e) solution of $\Phi(\chi, \varphi, \eta)$ at $\Upsilon=0.4$ of Example 2
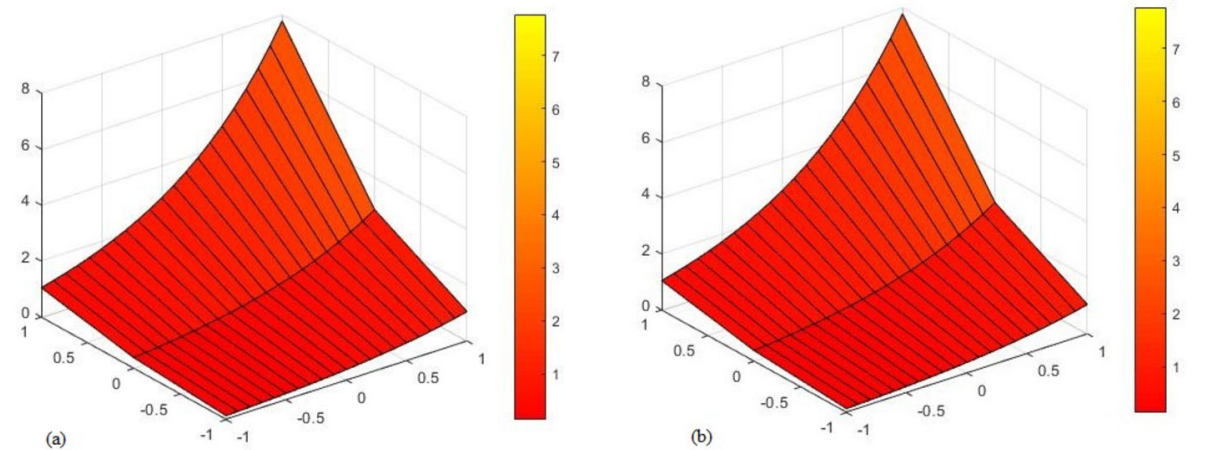

Figure 10 The graph of exact (a) and approximate (b) solution of $\Psi(\chi, \varphi, \eta)$ at $\Upsilon=1$ of Example 2

$\Upsilon=0.8,0.6$, and 0.4 for $\Phi$. In Fig. 10, we show the exact and analytical solution of $\Psi$ at $\Upsilon=1$. In Figs. 11 and 12 for different values of $\Upsilon=0.8,0.6$, and 0.4 for $\Psi$. The fractional results are investigated to be convergent to an integer-order result of each problem. 

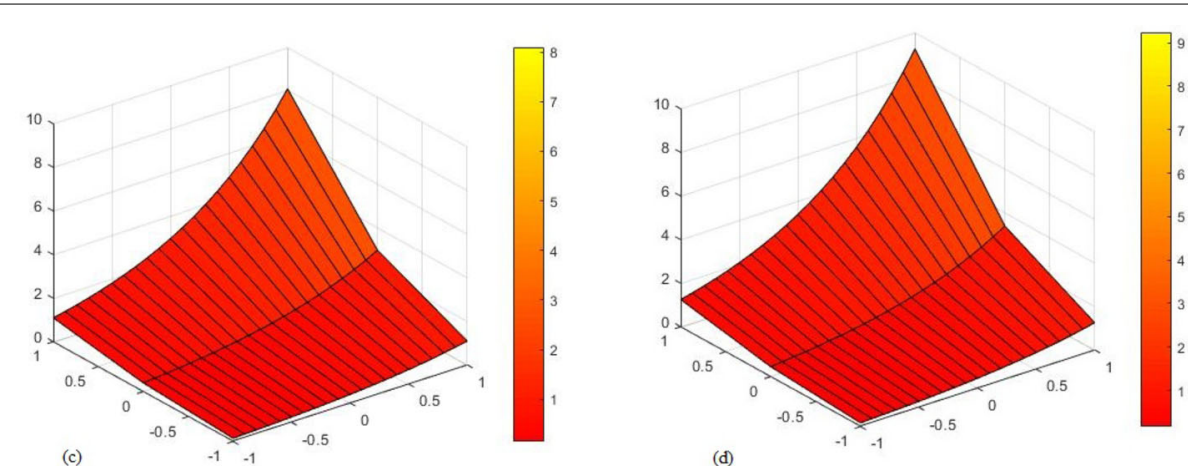

Figure 11 The graph of different approximate solution of $\Psi(\chi, \varphi, \eta)$ at $\Upsilon=(\mathbf{c}) 0.8$ and (d) 0.6 of Example 2

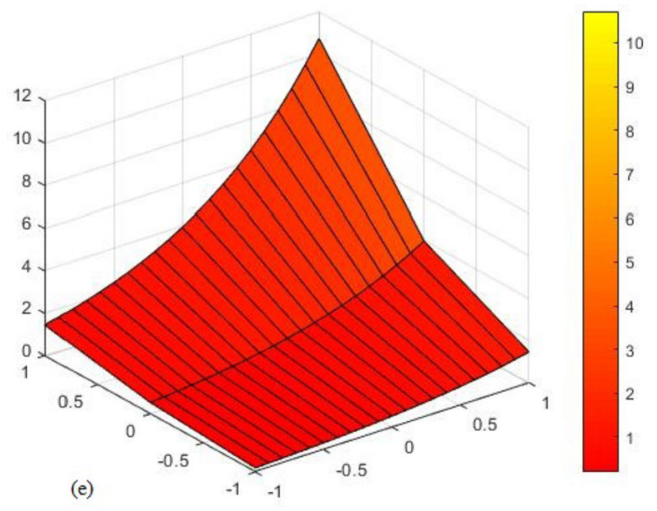

Figure 12 The graph of approximate (e) solution of $\Psi(\chi, \varphi, \eta)$ at $\Upsilon=0.4$ of Example 2

Example 3 Consider the time fractional-order $(2+1)$ dimensional Navier-Stokes equation

$$
\begin{aligned}
& D_{\eta}^{\Upsilon}(\Phi)+\Phi \frac{\partial \Phi}{\partial \chi}+\Psi \frac{\partial \Phi}{\partial \varphi}+\Theta \frac{\partial \Phi}{\partial \mathcal{Z}}=\rho\left[\frac{\partial^{2} \Phi}{\partial \chi^{2}}+\frac{\partial^{2} \Phi}{\partial \varphi^{2}}+\frac{\partial^{2} \Phi}{\partial \mathcal{Z}^{2}}\right]+q_{1}, \\
& D_{\eta}^{\Upsilon}(\Psi)+\Phi \frac{\partial \Psi}{\partial \chi}+\Psi \frac{\partial \Psi}{\partial \varphi}+\Theta \frac{\partial \Psi}{\partial \mathcal{Z}}=\rho\left[\frac{\partial^{2} \Psi}{\partial \chi^{2}}+\frac{\partial^{2} \Psi}{\partial \varphi^{2}}+\frac{\partial^{2} \Psi}{\partial \mathcal{Z}^{2}}\right]+q_{2}, \\
& D_{\eta}^{\Upsilon}(\Theta)+\Phi \frac{\partial \Theta}{\partial \chi}+\Psi \frac{\partial \Theta}{\partial \varphi}+\Theta \frac{\partial \Theta}{\partial \mathcal{Z}}=\rho\left[\frac{\partial^{2} \Theta}{\partial \chi^{2}}+\frac{\partial^{2} \Theta}{\partial \varphi^{2}}+\frac{\partial^{2} \Theta}{\partial \mathcal{Z}^{2}}\right]+q_{3},
\end{aligned}
$$

with the initial conditions

$$
\left\{\begin{array}{l}
\Phi(\chi, \varphi, \mathcal{Z}, 0)=-0.5 \chi+\varphi+\mathcal{Z} \\
\Psi(\chi, \varphi, \mathcal{Z}, 0)=\chi-0.5 \varphi+\mathcal{Z} \\
\Theta(\chi, \varphi, \mathcal{Z}, 0)=\chi+\varphi-0.5 \mathcal{Z}
\end{array}\right.
$$

Further, if $\rho$ is known, then $q_{1}=-\frac{1}{\rho} \frac{\partial g}{\partial \chi}, q_{2}=-\frac{1}{\rho} \frac{\partial g}{\partial \varphi}$, and $q_{1}=-\frac{1}{\rho} \frac{\partial g}{\partial \mathcal{Z}}$ can be determined. 
According to equation (7), the iteration formulas for equation (21) are as follows:

$$
\begin{aligned}
& \Phi_{m+1}(\chi, \varphi, \mathcal{Z}, \eta) \\
& =\Phi_{m}(\chi, \varphi, \mathcal{Z}, \eta)-L^{-1}\left[\frac { 1 } { s ^ { \Upsilon } } L \left\{s^{\Upsilon} \frac{\partial \Phi_{m}}{\partial \eta}\right.\right. \\
& \left.\left.\quad+\Phi_{m} \frac{\partial \Phi_{m}}{\partial \chi}+\Psi \frac{\partial \Phi_{m}}{\partial \varphi}+\Theta \frac{\partial \Phi_{m}}{\partial \mathcal{Z}}=\rho\left(\frac{\partial^{2} \Phi_{m}}{\partial \chi^{2}}+\frac{\partial^{2} \Phi_{m}}{\partial \varphi^{2}}+\frac{\partial^{2} \Phi_{m}}{\partial \mathcal{Z}^{2}}\right)+q_{1}\right\}\right] \\
& \Psi_{m+1}(\chi, \varphi, \mathcal{Z}, \eta) \\
& =\Psi_{m}(\chi, \varphi, \mathcal{Z}, \eta)-L^{-1}\left[\frac { 1 } { s ^ { \Upsilon } } L \left\{s^{\Upsilon} \frac{\partial \Psi_{m}}{\partial \eta}\right.\right. \\
& \left.\left.\quad+\Phi_{m} \frac{\partial \Psi_{m}}{\partial \chi}+\Psi_{m} \frac{\partial \Psi_{m}}{\partial \varphi}+\Theta \frac{\partial \Psi_{m}}{\partial \mathcal{Z}}=\rho\left(\frac{\partial^{2} \Psi_{m}}{\partial \chi^{2}}+\frac{\partial^{2} \Psi_{m}}{\partial \varphi^{2}}+\frac{\partial^{2} \Psi_{m}}{\partial \mathcal{Z}^{2}}\right)+q_{2}\right\}\right] \\
& \Theta_{m+1}(\chi, \varphi, \mathcal{Z}, \eta) \\
& =\Theta_{m}(\chi, \varphi, \mathcal{Z}, \eta)-L^{-1}\left[\frac { 1 } { s ^ { \Upsilon } } L \left\{s^{\Upsilon} \frac{\partial \Psi_{m}}{\partial \eta}\right.\right. \\
& \left.\left.\quad+\Phi_{m} \frac{\partial \Theta_{m}}{\partial \chi}+\Psi_{m} \frac{\partial \Theta_{m}}{\partial \varphi}+\Theta \frac{\partial \Theta_{m}}{\partial \mathcal{Z}}=\rho\left(\frac{\partial^{2} \Theta_{m}}{\partial \chi^{2}}+\frac{\partial^{2} \Theta_{m}}{\partial \varphi^{2}}+\frac{\partial^{2} \Theta_{m}}{\partial \mathcal{Z}^{2}}\right)+q_{3}\right\}\right]
\end{aligned}
$$

with the initial conditions

$$
\left\{\begin{array}{l}
\Phi(\chi, \varphi, \mathcal{Z}, 0)=-0.5 \chi+\varphi+\mathcal{Z} \\
\Psi(\chi, \varphi, \mathcal{Z}, 0)=\chi-0.5 \varphi+\mathcal{Z} \\
\Theta(\chi, \varphi, \mathcal{Z}, 0)=\chi+\varphi-0.5 \mathcal{Z}
\end{array}\right.
$$

Then $q_{1}, q_{2}$, and $q_{3}$ are equal to zero. For $m=0,1,2, \ldots$,

$$
\begin{aligned}
\Phi_{1}(\chi, \varphi, \mathcal{Z}, \eta)= & \Phi_{0}(\chi, \varphi, \mathcal{Z}, \eta)-L^{-1}\left[\frac { 1 } { s ^ { \Upsilon } } L \left\{s^{\Upsilon} \frac{\partial \Phi_{0}}{\partial \eta}+\Phi_{0} \frac{\partial \Phi_{0}}{\partial \chi}+\Psi_{0} \frac{\partial \Phi_{0}}{\partial \varphi}+\Theta_{0} \frac{\partial \Phi_{0}}{\partial \mathcal{Z}}\right.\right. \\
& \left.\left.+\rho\left(\frac{\partial^{2} \Phi_{0}}{\partial \chi^{2}}+\frac{\partial^{2} \Phi_{0}}{\partial \varphi^{2}}+\frac{\partial^{2} \Phi_{0}}{\partial \mathcal{Z}^{2}}\right)\right\}\right], \\
\Psi_{1}(\chi, \varphi, \mathcal{Z}, \eta)= & \Psi_{0}(\chi, \varphi, \mathcal{Z}, \eta)-L^{-1}\left[\frac { 1 } { s ^ { \Upsilon } } L \left\{s^{\Upsilon} \frac{\partial \Psi_{0}}{\partial \eta}+\Phi_{0} \frac{\partial \Psi_{0}}{\partial \chi}+\Psi_{0} \frac{\partial \Psi_{0}}{\partial \varphi}+\Theta_{0} \frac{\partial \Psi_{0}}{\partial \mathcal{Z}}\right.\right. \\
& \left.\left.+\rho\left(\frac{\partial^{2} \Psi_{0}}{\partial \chi^{2}}+\frac{\partial^{2} \Psi_{0}}{\partial \varphi^{2}}+\frac{\partial^{2} \Psi_{0}}{\partial \mathcal{Z}^{2}}\right)\right\}\right], \\
\Theta_{1}(\chi, \varphi, \mathcal{Z}, \eta)= & \Theta_{0}(\chi, \varphi, \mathcal{Z}, \eta)-L^{-1}\left[\frac { 1 } { s ^ { \Upsilon } } L \left\{s^{\Upsilon} \frac{\partial \Psi_{0}}{\partial \eta}+\Phi_{0} \frac{\partial \Theta_{0}}{\partial \chi}+\Psi_{0} \frac{\partial \Theta_{0}}{\partial \varphi}+\Theta_{0} \frac{\partial \Theta_{0}}{\partial \mathcal{Z}}\right.\right. \\
& \left.\left.+\rho\left(\frac{\partial^{2} \Theta_{0}}{\partial \chi^{2}}+\frac{\partial^{2} \Theta_{0}}{\partial \varphi^{2}}+\frac{\partial^{2} \Theta_{0}}{\partial \mathcal{Z}^{2}}\right)\right\}\right], \\
\Phi_{1}(\chi, \varphi, \mathcal{Z}, \eta)= & -0.5 \chi+\varphi+\mathcal{Z}-\frac{2.25 \chi \eta^{\Upsilon}}{\Gamma(\Upsilon+1)}, \\
\Psi_{1}(\chi, \varphi, \mathcal{Z}, \eta)=\chi & -0.5 \varphi+\mathcal{Z}-\frac{2.25 \varphi \eta^{\Upsilon}}{\Gamma(\Upsilon+1)},
\end{aligned}
$$


$\Theta_{1}(\chi, \varphi, \mathcal{Z}, \eta)=\chi+\varphi-0.5 \mathcal{Z}-\frac{2.25 \mathcal{Z} \eta^{\Upsilon}}{\Gamma(\Upsilon+1)}$,

$\Phi_{2}(\chi, \varphi, \mathcal{Z}, \eta)=\Phi_{1}(\chi, \varphi, \mathcal{Z}, \eta)-L^{-1}\left[\frac{1}{s^{\Upsilon}} L\left\{s^{\Upsilon} \frac{\partial \Phi_{1}}{\partial \eta}+\Phi_{1} \frac{\partial \Phi_{1}}{\partial \chi}+\Psi_{1} \frac{\partial \Phi_{1}}{\partial \varphi}+\Theta_{1} \frac{\partial \Phi_{1}}{\partial \mathcal{Z}}\right.\right.$

$$
\left.\left.+\rho\left(\frac{\partial^{2} \Phi_{1}}{\partial \chi^{2}}+\frac{\partial^{2} \Phi_{1}}{\partial \varphi^{2}}+\frac{\partial^{2} \Phi_{1}}{\partial \mathcal{Z}^{2}}\right)\right\}\right]
$$

$\Psi_{2}(\chi, \varphi, \mathcal{Z}, \eta)=\Psi_{1}(\chi, \varphi, \mathcal{Z}, \eta)-L^{-1}\left[\frac{1}{s^{\Upsilon}} L\left\{s^{\Upsilon} \frac{\partial \Psi_{1}}{\partial \eta}+\Phi_{1} \frac{\partial \Psi_{1}}{\partial \chi}+\Psi_{1} \frac{\partial \Psi_{1}}{\partial \varphi}+\Theta_{1} \frac{\partial \Psi_{1}}{\partial \mathcal{Z}}\right.\right.$

$$
\left.\left.+\rho\left(\frac{\partial^{2} \Psi_{1}}{\partial \chi^{2}}+\frac{\partial^{2} \Psi_{1}}{\partial \varphi^{2}}+\frac{\partial^{2} \Psi_{1}}{\partial \mathcal{Z}^{2}}\right)\right\}\right]
$$

$\Theta_{2}(\chi, \varphi, \mathcal{Z}, \eta)=\Theta_{1}(\chi, \varphi, \mathcal{Z}, \eta)-L^{-1}\left[\frac{1}{s^{\Upsilon}} L\left\{s^{\Upsilon} \frac{\partial \Psi_{1}}{\partial \eta}+\Phi_{1} \frac{\partial \Theta_{1}}{\partial \chi}+\Psi_{1} \frac{\partial \Theta_{1}}{\partial \varphi}+\Theta_{1} \frac{\partial \Theta_{1}}{\partial \mathcal{Z}}\right.\right.$

$$
\left.\left.+\rho\left(\frac{\partial^{2} \Theta_{1}}{\partial \chi^{2}}+\frac{\partial^{2} \Theta_{1}}{\partial \varphi^{2}}+\frac{\partial^{2} \Theta_{1}}{\partial \mathcal{Z}^{2}}\right)\right\}\right]
$$

$\Phi_{2}(\chi, \varphi, \mathcal{Z}, \eta)=-0.5 \chi+\varphi+\mathcal{Z}-\frac{2.25 \chi \eta^{\Upsilon}}{\Gamma(\Upsilon+1)}+\frac{2(2.25) \chi \eta^{2 \Upsilon}}{\Gamma(2 \Upsilon+1)}(-0.5 \chi+\varphi+\mathcal{Z}$,

$\Psi_{2}(\chi, \varphi, \mathcal{Z}, \eta)=\chi-0.5 \varphi+\mathcal{Z}-\frac{2.25 \varphi \eta^{\Upsilon}}{\Gamma(\Upsilon+1)}+\frac{2(2.25) \varphi \eta^{2 \Upsilon}}{\Gamma(2 \Upsilon+1)}(\chi-0.5 \varphi+\mathcal{Z})$,

$\Theta_{2}(\chi, \varphi, \mathcal{Z}, \eta)=\chi+\varphi-0.5 \mathcal{Z}-\frac{2.25 \mathcal{Z} \eta^{\Upsilon}}{\Gamma(\Upsilon+1)}+\frac{2(2.25) \mathcal{Z} \eta^{2 \Upsilon}}{\Gamma(2 \Upsilon+1)}(\chi+\varphi-0.5 \mathcal{Z})$,

$\Phi_{3}(\chi, \varphi, \mathcal{Z}, \eta)=\Phi_{2}(\chi, \varphi, \mathcal{Z}, \eta)-L^{-1}\left[\frac{1}{s^{\Upsilon}} L\left\{s^{\Upsilon} \frac{\partial \Phi_{2}}{\partial \eta}+\Phi_{2} \frac{\partial \Phi_{2}}{\partial \chi}+\Psi_{2} \frac{\partial \Phi_{2}}{\partial \varphi}+\Theta_{2} \frac{\partial \Phi_{2}}{\partial \mathcal{Z}}\right.\right.$

$$
\left.\left.+\rho\left(\frac{\partial^{2} \Phi_{2}}{\partial \chi^{2}}+\frac{\partial^{2} \Phi_{2}}{\partial \varphi^{2}}+\frac{\partial^{2} \Phi_{2}}{\partial \mathcal{Z}^{2}}\right)\right\}\right]
$$

$\Psi_{3}(\chi, \varphi, \mathcal{Z}, \eta)=\Psi_{2}(\chi, \varphi, \mathcal{Z}, \eta)-L^{-1}\left[\frac{1}{s^{\Upsilon}} L\left\{s^{\Upsilon} \frac{\partial \Psi_{2}}{\partial \eta}+\Phi_{2} \frac{\partial \Psi_{2}}{\partial \chi}+\Psi_{2} \frac{\partial \Psi_{2}}{\partial \varphi}+\Theta_{2} \frac{\partial \Psi_{2}}{\partial \mathcal{Z}}\right.\right.$

$$
\left.\left.+\rho\left(\frac{\partial^{2} \Psi_{2}}{\partial \chi^{2}}+\frac{\partial^{2} \Psi_{2}}{\partial \varphi^{2}}+\frac{\partial^{2} \Psi_{2}}{\partial \mathcal{Z}^{2}}\right)\right\}\right]
$$

$\Theta_{3}(\chi, \varphi, \mathcal{Z}, \eta)=\Theta_{2}(\chi, \varphi, \mathcal{Z}, \eta)-L^{-1}\left[\frac{1}{s^{\Upsilon}} L\left\{s^{\Upsilon} \frac{\partial \Psi_{2}}{\partial \eta}+\Phi_{2} \frac{\partial \Theta_{2}}{\partial \chi}+\Psi_{2} \frac{\partial \Theta_{2}}{\partial \varphi}+\Theta_{2} \frac{\partial \Theta_{2}}{\partial \mathcal{Z}}\right.\right.$

$$
\left.\left.+\rho\left(\frac{\partial^{2} \Theta_{2}}{\partial \chi^{2}}+\frac{\partial^{2} \Theta_{2}}{\partial \varphi^{2}}+\frac{\partial^{2} \Theta_{2}}{\partial \mathcal{Z}^{2}}\right)\right\}\right] \text {, }
$$

$\Phi_{3}(\chi, \varphi, \mathcal{Z}, \eta)=-0.5 \chi+\varphi+\mathcal{Z}-\frac{2.25 \chi \eta^{\Upsilon}}{\Gamma(\Upsilon+1)}+\frac{2(2.25) \chi \eta^{2 \Upsilon}}{\Gamma(2 \Upsilon+1)}$

$$
\times(-0.5 \chi+\varphi+\mathcal{Z})-\frac{(2.25)^{2} \chi\left(4(\Gamma(\Upsilon+1))^{2}+\Gamma(2 \Upsilon+1)\right) \eta^{3 \Upsilon}}{\Gamma(2 \Upsilon+1)(\Gamma(\Upsilon+1))^{2}},
$$

$\Psi_{3}(\chi, \varphi, \mathcal{Z}, \eta)=\chi-0.5 \varphi+\mathcal{Z}-\frac{2.25 \varphi \eta^{\Upsilon}}{\Gamma(\Upsilon+1)}+\frac{2(2.25) \varphi \eta^{2 \Upsilon}}{\Gamma(2 \Upsilon+1)}$

$$
\times(\chi-0.5 \varphi+\mathcal{Z})-\frac{(2.25)^{2} \varphi\left(4(\Gamma(\Upsilon+1))^{2}+\Gamma(2 \Upsilon+1)\right) \eta^{3 \Upsilon}}{\Gamma(2 \Upsilon+1)(\Gamma(\Upsilon+1))^{2}},
$$




$$
\begin{aligned}
\Theta_{3}(\chi, \varphi, \mathcal{Z}, \eta)= & \chi+\varphi-0.5 \mathcal{Z}-\frac{2.25 \mathcal{Z} \eta^{\Upsilon}}{\Gamma(\Upsilon+1)}+\frac{2(2.25) \mathcal{Z} \eta^{2 \Upsilon}}{\Gamma(2 \Upsilon+1)} \\
& \times(\chi+\varphi-0.5 \mathcal{Z})-\frac{(2.25)^{2} \mathcal{Z}\left(4(\Gamma(\Upsilon+1))^{2}+\Gamma(2 \Upsilon+1)\right) \eta^{3 \Upsilon}}{\Gamma(2 \Upsilon+1)(\Gamma(\Upsilon+1))^{2}} .
\end{aligned}
$$

In the same procedure, the remaining $\Phi_{m}, \Psi_{m}$, and $\Theta_{m}(m>3)$ components of the VITM solution can be obtained smoothly.

$$
\begin{aligned}
\Phi(\chi, \varphi, \mathcal{Z}, \eta)= & -0.5 \chi+\varphi+\mathcal{Z}-\frac{2.25 \chi \eta^{\Upsilon}}{\Gamma(\Upsilon+1)}+\frac{2(2.25) \chi \eta^{2 \Upsilon}}{\Gamma(2 \Upsilon+1)} \\
& \times(-0.5 \chi+\varphi+\mathcal{Z})-\frac{(2.25)^{2} \chi\left(4(\mathcal{Z}(\Upsilon+1))^{2}+\Gamma(2 \Upsilon+1)\right) \eta^{3 \Upsilon}}{\Gamma(2 \Upsilon+1)(\Gamma(\Upsilon+1))^{2}}+\cdots, \\
\Psi(\chi, \varphi, \mathcal{Z}, \eta)= & \chi-0.5 \varphi+\mathcal{Z}-\frac{2.25 \varphi \eta^{\Upsilon}}{\Gamma(\Upsilon+1)}+\frac{2(2.25) \varphi \eta^{2 \Upsilon}}{\Gamma(2 \Upsilon+1)} \\
& \times(\chi-0.5 \varphi+\mathcal{Z})-\frac{(2.25)^{2} \varphi\left(4(\Gamma(\Upsilon+1))^{2}+\Gamma(2 \Upsilon+1)\right) \eta^{3 \Upsilon}}{\Gamma(2 \Upsilon+1)(\Gamma(\Upsilon+1))^{2}}+\cdots, \\
\Theta(\chi, \varphi, \mathcal{Z}, \eta)= & \chi+\varphi-0.5 \mathcal{Z}-\frac{2.25 \mathcal{Z} \eta^{\Upsilon}}{\Gamma(\Upsilon+1)}+\frac{2(2.25) \mathcal{Z} \eta^{2 \Upsilon}}{\Gamma(2 \Upsilon+1)} \\
& \times(\chi+\varphi-0.5 \mathcal{Z})-\frac{(2.25)^{2} \mathcal{Z}\left(4(\Gamma(\Upsilon+1))^{2}+\Gamma(2 \Upsilon+1)\right) \eta^{3 \Upsilon}}{\Gamma(2 \Upsilon+1)(\Gamma(\Upsilon+1))^{2}}+\cdots
\end{aligned}
$$

The exact result of equation (21) at $\Upsilon=1$ and $q_{1}=q_{2}=q_{3}=0$ is as follows:

$$
\begin{aligned}
& \Phi(\chi, \varphi, \mathcal{Z}, \eta)=\frac{-0.5 \chi+\varphi+\mathcal{Z}-2.25 \chi \eta}{1-2.25 \eta^{2}} \\
& \Psi(\chi, \varphi, \mathcal{Z}, \eta)=\frac{\chi-0.5 \varphi+\mathcal{Z}-2.25 \varphi \eta}{1-2.25 \eta^{2}} \\
& \Theta(\chi, \varphi, \mathcal{Z}, \eta)=\frac{\chi+\varphi-0.5 \mathcal{Z}-2.25 \mathcal{Z} \eta}{1-2.25 \eta^{2}}
\end{aligned}
$$

Figures 13 and 14 show the behavior of solutions of the exact and analytical results using the initial conditions given in equation (22). In Fig. 13 (a) of $\Phi$, subfigure (b) of $\Psi$, and Fig. 14 of $\Theta$ the exact and analytical solutions at $\Upsilon=1$ show close contact with each other.

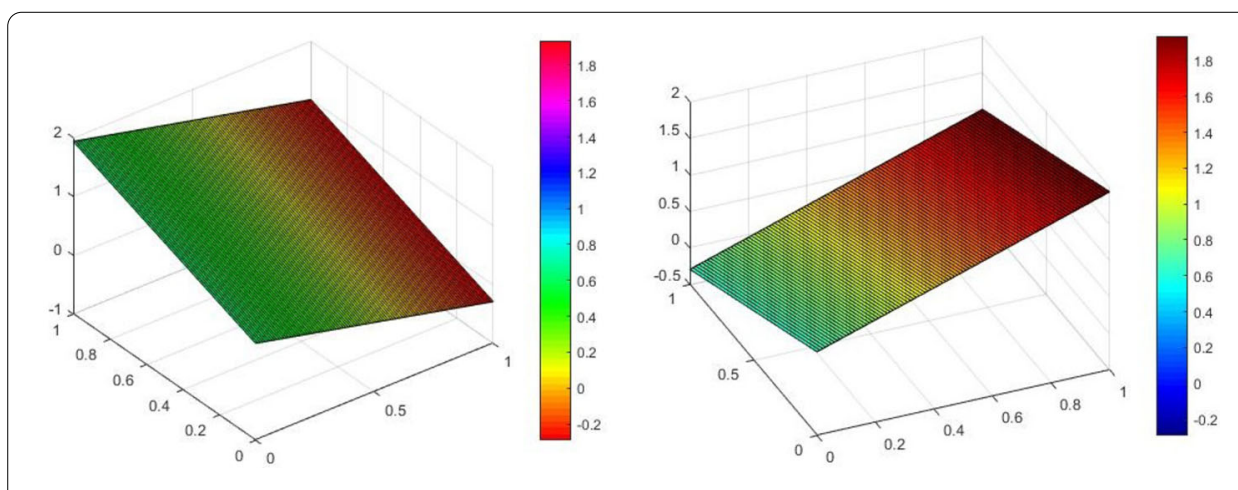

Figure 13 The (a) graph of exact and approximate solution of $\Phi(\chi, \varphi, \eta)(\mathbf{b}) \Psi(\chi, \varphi, \eta)$ at $\Upsilon=1$ of Example 3 


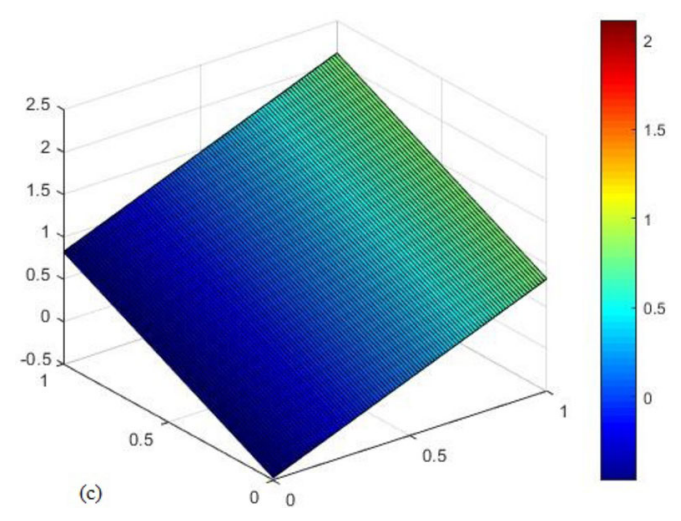

Figure 14 The graph of exact and approximate solution of $\Theta(\chi, \varphi, \eta)$ at $\Upsilon=1$ of Example 3

\section{Conclusion}

In this article, we evaluated the fractional-order multi-dimensional Navier-Stokes equations using a variational iteration transform technique. The solutions for specific examples are explained using the current method. The VITM result is in close contact with the exact result of the given problems. The graphical analysis of the fractional-order solutions obtained has verified the convergence towards the solutions of integer order. One may see that the obtained results are in excellent agreement with FRDTM [54] and HPETM [48]. Moreover, the present technique is straightforward, simple, and carrying less computational cost; the suggested method can be modified to solve other fractional-order partial differential equations.

\section{Acknowledgements}

This research was supported by the Basic Science Research Program through the National Research Foundation of Korea (NRF) funded by the Ministry of Education (No. 2017R1D1A1B05030422).

\section{Funding}

Not applicable.

\section{Availability of data and materials}

Not applicable.

\section{Competing interests}

The authors declare that they have no competing interests.

\section{Authors' contributions}

All authors have equally contributed. All authors read and approved the final manuscript.

\section{Author details}

${ }^{1}$ Department of Mathematics, Huzhou University, Huzhou 313000, P.R. China. ${ }^{2}$ Hunan Provincial Key Laboratory of Mathematical Modeling and Analysis in Engineering, Changsha University of Science \& Technology, Changsha 410114 , P.R. China. Informetrics Research Group, Ton Duc Thang University, Ho Chi Minh City, Vietnam. ${ }^{4}$ Faculty of Mathematics \& Statistics, Ton Duc Thang University, Ho Chi Minh City, Vietnam. ${ }^{5}$ Department of Mathematics, Anand International College of Engineering, Jaipur, India. ${ }^{6}$ Department of Mechanical Engineering, Sejong University, Seoul 05006, Korea.

\section{Publisher's Note}

Springer Nature remains neutral with regard to jurisdictional claims in published maps and institutional affiliations.

Received: 1 October 2020 Accepted: 19 January 2021 Published online: 30 January 2021

\section{References}

1. Taylor, G.l.: On the decay of vortices in a viscous fluid. Philos. Mag. 46, 671-674 (1923)

2. Ross, B.: A brief history and exposition of the fundamental theory of fractional calculus. In: Ross, B. (ed.) The Fractional Calculus and Its Applications, pp. 1-36. Springer, Berlin (1975) 
3. Miller, K.S., Ross, B.: An Introduction to the Fractional Calculus and Fractional Differential Equation. Wiley, New York (1993)

4. Kilbas, A.A., Srivastava, H.M., Trujillo, J.J.: Theory and Application of Fractional Differential Equations. Elsevier, Amsterdam (2006)

5. Kumar, S., Nisar, K.S., Kumar, R., Cattani, C., Samet, B.: A new Rabotnov fractional-exponential function based fractional derivative for diffusion equation under external force. Math. Methods Appl. Sci. 43(7), 4460-4471 (2020)

6. Kumar, S., Ghosh, S., Samet, B., Goufo, E.F.D.: An analysis for heat equations arises in diffusion process using new Yang Abdel Aty Cattani fractional operator. Math. Methods Appl. Sci. 43(9), 6062-6080 (2020)

7. Kumar, S., Kumar, R., Agarwal, R.P., Samet, B.: A study of fractional Lotka-Volterra population model using Haar wavelet and Adams-Bash forth-Moulton methods. Math. Methods Appl. Sci. 43(8), 5564-5578 (2020)

8. Kumar, S.: A new analytical modelling for fractional telegraph equation via Laplace transform. Appl. Math. Model. 38(13), 3154-3163 (2014)

9. Kumar, S., Rashidi, M.M.: New analytical method for gas dynamics equation arising in shock fronts. Comput. Phys. Commun. 185(7), 1947-1954 (2014)

10. Kumar, S., Kumar, A., Baleanu, D.: Two analytical methods for time-fractional nonlinear coupled Boussinesq-Burgers equations arise in propagation of shallow water waves. Nonlinear Dyn. 85(2), 699-715 (2016)

11. Kumar, S., Kumar, D., Abbasbandy, S., Rashidi, M.M.: Analytical solution of fractional Navier-Stokes equation by using modified Laplace decomposition method. Ain Shams Eng. J. 5(2), 569-574 (2014)

12. Wang, K.-L., Yao, S.-W., Liu, Y.-P., Zhang, L.-N.: A fractal variational principle for the telegraph equation with fractal derivatives. Fractals 28(4), 2050058 (2020)

13. Wang, K.-L., Wang, K.-J., He, C.-H.: Physical insight of local fractional calculus and its application to fractional Kdv-Burgers-Kuramoto equation. Fractals 27(07), 1950122 (2019)

14. LV, C., Zhou, H., Deng, F.: Mittag-Leffler stabilization of an unstable time fractional hyperbolic PDE. IEEE Access 7, 102580-102588 (2019)

15. Shoaib, M., Abdeljawad, T., Sarwar, M., Jarad, F.: Fixed point theorems for multi-valued contractions in b-metric spaces with applications to fractional differential and integral equations. IEEE Access 7, 127373-127383 (2019)

16. Jena, R.M., Chakraverty, S., Baleanu, D., Alqurashi, M.M.: New aspects of ZZ transform to fractional operators with Mittag-Leffler kernel. Front. Phys. 8, 352 (2020)

17. Jena, R.M., Chakraverty, S., Yavuz, M.: Two-hybrid techniques coupled with an integral transform for Caputo time-fractional Navier-Stokes equations. Prog. Fract. Differ. Appl. 6(4), 201-213 (2020)

18. Chakraverty, S., Jena, R.M., Jena, S.K.: Time-fractional order biological systems with uncertain parameters. Synth. Lect. Math. Stat. 12(1), 1-160 (2020)

19. Jena, R.M., Chakraverty, S., Baleanu, D.: Solitary wave solution for a generalized Hirota-Satsuma coupled KdV and MKdV equations: a semi-analytical approach. Alex. Eng. J. (2020). To appear

20. Jena, R.M., Chakraverty, S.: Q-homotopy analysis Aboodh transform method based solution of proportional delay time-fractional partial differential equations. J. Interdiscip. Math. 22(6), 931-950 (2019)

21. Srivastava, H.M., Jena, R.M., Chakraverty, S., Jena, S.K.: Dynamic response analysis of fractionally-damped generalized Bagley-Torvik equation subject to external loads. Russ. J. Math. Phys. 27, 254-268 (2020)

22. Jena, R.M., Chakraverty, S., Baleanu, D.: On the solution of an imprecisely defined nonlinear time-fractional dynamical model of marriage. Mathematics 7(8), 689 (2019)

23. Jena, R.M., Chakraverty, S., Jena, S.K.: Dynamic response analysis of fractionally damped beams subjected to external loads using homotopy analysis method. J. Appl. Comput. Mech. 5(2), 355-366 (2019)

24. Jena, R.M., Chakraverty, S.: Residual power series method for solving time-fractional model of vibration equation of large membranes. J. Appl. Comput. Mech. 5(4), 603-615 (2019)

25. Wang, Q.: Numerical solutions for fractional KdV-Burgers equation by Adomian decomposition method. Appl. Math. Comput. 182(2), 1048-1055 (2006)

26. Momani, S., Shawagfeh, N.T.: Decomposition method for solving fractional Riccati differential equations. Appl. Math. Comput. 182(2), 1083-1092 (2006)

27. Gejii, V.D., Jafari, H.: Solving a multi-order fractional differential equation. Appl. Math. Comput. 189(1), 541-548 (2007)

28. Inc, M.: The approximate and exact solutions of the space and time-fractional Burgers equations with initial conditions by variational iteration method. J. Math. Anal. Appl. 345(1), 476-484 (2008)

29. Odibat, Z., Momani, S.: Modified homotopy perturbation method: application to quadratic Riccati differential equation of fractional order. Chaos Solitons Fractals 36(1), 167-174 (2008)

30. Hosseinnia, S., Ranjbar, A., Momani, S.: Using an enhanced homotopy perturbation method in fractional differential equations via deforming the linear part. Comput. Math. Appl. 56(12), 3138-3149 (2008)

31. Dhaigude, C.D., Nikam, V.R.: Solution of fractional partial differential equations using iterative method. Fract. Calc. Appl. Anal. 15(4), 684-699 (2012)

32. Secer, A., Akinlar, M.A., Cevikel, A.: Efficient solutions of systems of fractional partial differential equations by the differential transform method. Adv. Differ. Equ. 2013, 188 (2012)

33. Yang, X.J., Baleanu, D.: Fractal heat conduction problem solved by local fractional variation iteration method. Therm. Sci. 17(2), 625-628 (2013)

34. Yang, X.J., Baleanu, D., Khan, Y., Mohyud-Din, S.T.: Local fractional variational iteration method for differential and wave equations on Cantor sets. Rom. J. Phys. 59(1-2), 36-48 (2014)

35. Baleanu, D., Machado, J.A.T., Cattani, C., Baleanu, M.C., Yang, X.J.: Local fractional variational iteration and decomposition methods for wave equation on Cantor sets within local fractional operators. Abstr. Appl. Anal. 2014, 535048 (2014)

36. Zainal, N.H., KIman, A.: Solving fractional partial differential equations with corrected Fourier series method. Abstr. Appl. Anal. 2014, 958931 (2014)

37. He, J.H.: Approximate analytical solution for seepage flow with fractional derivatives in porous media. Comput. Methods Appl. Mech. Eng. 167(1-2), 57-68 (1998)

38. Geng, F., Lin, Y., Cui, M.: A piecewise variational iteration method for Riccati differential equations. Comput. Math. Appl. 58(11-12), 2518-2522 (2009) 
39. Odibat, Z., Momani, S.: The variational iteration method: an efficient scheme for handling fractional partial differential equations in fluid mechanics. Comput. Math. Appl. 58(11-12), 2199-2208 (2009)

40. Odibat, Z.M.: A study on the convergence of variational iteration method. Math. Comput. Model. 51(9-10), 1181-1192 (2010)

41. Yang, X.J., Baleanu, D., Khan, Y., Mohyud-Din, S.T.: Local fractional variational iteration method for diffusion and wave equations on Cantor sets. Rom. J. Phys. 59(1-2), 36-48 (2014)

42. Prakash, A., Kumar, M., Sharma, K.K.: Numerical method for solving fractional coupled Burgers equations. Appl. Math. Comput. 260, 314-320 (2015)

43. Sakar, M.G., Ergoren, H.: Alternative variational iteration method for solving the time-fractional Fornberg-Whitham equation. Appl. Math. Model. 39(14), 3972-3979 (2015)

44. Yu, J., Tan, L., Zhou, S., Wang, L., Siddique, M.A.: Image denoising algorithm based on entropy and adaptive fractional order calculus operator. IEEE Access 5, 12275-12285 (2017)

45. Singh, B.K., Kumar, P.: Fractional variational iteration method for solving fractional partial differential equations with proportional delay. Int. J. Differ. Equ. 2017, 5206380 (2017)

46. Bistafa, S.R.: On the development of the Navier-Stokes equation by Navier. Rev. Bras. Ensino Fasica 40(2), e2603 (2018)

47. Jaber, K.K., Ahmad, R.S.: Analytical solution of the time fractional Navier-Stokes equation. Ain Shams Eng. J. 9(4), 1917-1927 (2018)

48. Jena, R.M., Chakraverty, S.: Solving time-fractional Navier-Stokes equations using homotopy perturbation Elzaki transform. SN Appl. Sci. 1(1), 16 (2019)

49. Akbar, T., Zia, Q.M.Z.: Some exact solutions of two-dimensional Navier-Stokes equations by generalizing the local vorticity. Adv. Mech. Eng. 11(4), 1687814019831893 (2019)

50. Dubey, V.P., Kumar, R., Kumar, D., Khan, I., Singh, J.: An efficient computational scheme for nonlinear time fractional systems of partial differential equations arising in physical sciences. Adv. Differ. Equ. 2020(1), 46 (2020)

51. Duan, X., Dang, Y., Lu, J.: A variational level set method for topology optimization problems in Navier-Stokes flow. IEEE Access 8, 48697-48706 (2020)

52. Odibat, Z.M.: A study on the convergence of variational iteration method. Math. Comput. Model. 51(9-10), 1181-1192 (2010)

53. Zedan, H.A., Tantawy, S.S., Sayed, Y.M.: Convergence of the variational iteration method for initial-boundary value problem of fractional integro-differential equations. J. Fract. Calc. Appl. 5(supplement 3), 1-14 (2014)

54. Singh, B.K., Kumar, P.: FRDTM for numerical simulation of multi-dimensional, time-fractional model of Navier-Stokes equation. Ain Shams Eng. J. 9(4), 827-834 (2018)

\section{Submit your manuscript to a SpringerOpen ${ }^{\circ}$ journal and benefit from:}

- Convenient online submission

- Rigorous peer review

- Open access: articles freely available online

- High visibility within the field

- Retaining the copyright to your article

Submit your next manuscript at $\boldsymbol{\nabla}$ springeropen.com 